\title{
Monitoramento a usuários pós-AVC na Atenção Primária: uma revisão sistemática
}

\author{
Monitoring of post-stroke users in Primary Care: \\ a systematic review
}

\section{Seguimiento de usuarios post-ictus en Atención Primaria: una revisión sistemática}

\author{
Laura Faustino Gonçalves, Karina Mary De Paiva², Patrícia Haas ${ }^{3}$ \\ 1.Universidade Federal de Santa Catarina - UFSC; Departamento de Fonoaudiologia. Florianópolis-SC, \\ Brasil. Ordic: https://orcid.org/0000-0002-0043-4349 \\ 2.Professora Doutora do Curso de Fonoaudiologia pela Universidade Federal de Santa Catarina - UFSC. \\ Florianópolis-SC, Brasil. Orcid: https://orcid.org/0000-0001-7086-534X \\ 3.Universidade Federal de Santa Catarina - UFSC; Departamento de Fonoaudiologia. Florianópolis-SC, \\ Brasil. Orcid: https://orcid.org/0000-0001-9797-7755
}

\begin{abstract}
Resumo
Introdução. Aproximadamente $60 \%$ dos pacientes ficam com sequelas motoras, cognitivas e distúrbios de comunicação. Objetivo. Apresentar evidências científicas com base em revisão sistemática da literatura achados referentes ao monitoramento de usuários pós-AVC na atenção primária. Método. Para a seleção dos estudos foi utilizada a combinação baseada no Medical Subject Heading Terms (MeSH). Foram utilizadas as bases de dados MEDLINE (Pubmed), LILACS, SciELO, SCOPUS, WEB OF SCIENCE e BIREME. Sem restrição de idioma, período e localização. Resultados. Foram identificados 88 artigos inicialmente, dos quais 25 foram para a avaliação de resumos. Desses, 22 foram excluídos por não responderem à pergunta norteadora. Procedeu-se a leitura na íntegra dos 2 artigos e após a aplicação dos critérios de elegibilidade, no qual foram inclusos na pesquisa. Conclusão. Os estudos mostraram uma evolução significativa dos pacientes que realizaram acompanhamento na atenção primária pós-AVC, demonstrando assim a sua importância de continuidade de intervenção após a alta hospitalar.
\end{abstract}

Unitermos. Acidente Vascular Cerebral; Atenção Primária à Saúde; Infarto Cerebral

\begin{abstract}
Introduction. Approximately $60 \%$ of patients have motor, cognitive, and communication disorders. Objective. To present scientific evidence based on a systematic review of the literature findings related to the attention (or monitoring) to post-stroke users. Method. For the selection of studies, the combination based on the Medical Subject Heading Terms (MeSH) was used. The MEDLINE (Pubmed), LILACS, SciELO, SCOPUS, WEB OF SCIENCE, and BIREME databases were used. No language, period, and location restrictions. Results. 88 articles were initially identified, of which 25 were for the evaluation of abstracts. Of these, 22 were excluded for not answering the guiding question. The two articles were read in full and after the application of the eligibility criteria, in which they were included in the research. Conclusion. The studies showed a significant evolution of patients who underwent follow-up in post-stroke primary care, thus demonstrating the importance of continuing the intervention after hospital discharge.
\end{abstract}

Keywords. Stroke; Primary Health Care; Cerebral Infarction

\section{Resumen}

Introducción. Aproximadamente el $60 \%$ de los pacientes presentan trastornos motores, cognitivos y de la comunicación. Objetivo. Presentar evidencia científica basada en una revisión sistemática de los hallazgos de la literatura relacionados con la atención (o 
seguimiento) a los usuarios post-ictus. Método. Para la selección de estudios se utilizó la combinación basada en los Medical Subject Heading Terms (MeSH). Se utilizaron las bases de datos MEDLINE (Pubmed), LILACS, SciELO, SCOPUS, WEB OF SCIENCE y BIREME. Sin restricción de idioma, período y ubicación. Resultados. Se identificaron inicialmente 88 artículos, de los cuales 25 fueron para la evaluación de resúmenes. De estos, 22 fueron excluidos por no responder a la pregunta orientadora. Los dos artículos fueron leídos íntegramente y previa aplicación de los criterios de elegibilidad, en los que fueron incluidos en la investigación. Conclusión. Los estudios evidenciaron una evolución significativa de los pacientes a los que se les realizó seguimiento en atención primaria post-ictus, demostrando así la importancia de la continuidad de la intervención tras el alta hospitalaria.

Palabras clave. Accidente cerebrovascular; Atención Primaria; Infarto cerebral

Trabalho realizado na Universidade Federal de Santa Catarina - UFSC. Florianópolis-SC, Brasil.

\section{INTRODUÇÃO}

O Acidente Vascular Cerebral (AVC) é relatado como um déficit neurológico decorrente da interrupção do fluxo sanguíneo na região encefálica, causando diminuição do suprimento neuronal e morte de células nervosas ${ }^{1}$. O AVC pode ser classificado como isquêmico ( $\mathrm{AVCi}$ ), pela oclusão de algum vaso sanguíneo para o cérebro, causando um coágulo ou trombo que interrompe o fluxo do sangue e dificultando o fornecimento de oxigênio e substratos para tecido cerebral; ou hemorrágico (AVCh), quando há uma ruptura no vaso sanguíneo e consequentemente efusão de sangue na região². O primeiro tipo é o mais frequente, representando 80 a $85 \%$ dos $\operatorname{casos}^{3}$.

Os fatores de risco para o AVC envolvem fatores modificáveis e não modificáveis; sendo estes últimos relacionados a questões genéticas, apresentando risco mais elevado no sexo masculino e idade superior a 70 anos $^{4}$. Tendo em vista que os fatores de risco modificáveis, 
envolvem o estilo de vida (alimentação, atividade física e hábitos de risco), assim como o tabagismo e o consumo de álcool, destaca-se a importância destas ações na atenção aos usuários. Além disso, salienta-se também algumas doenças crônicas, como Hipertensão Arterial Sistêmica (HAS), colesterol alto, diabetes e problemas cardíacos, as quais remetem à necessidade do monitoramento constante ${ }^{5}$.

No Brasil, a Linha de Cuidado ao AVC instituída em 2012, abrange ações com o objetivo principal de redução da morbimortalidade, contemplando o tratamento desde o evento agudo, visando a redução de danos, até os programas de reabilitação ambulatoriais e domiciliares, minimizando limitações. Aproximadamente $60 \%$ dos pacientes ficam com sequelas motoras, cognitivas e distúrbios de comunicação. O monitoramento pós AVC representa uma importante estratégia de continuidade do cuidado e deve ser articulado pela atenção primária, como tentativa de mudar o panorama atual com queda do seguimento terapêutico após a alta hospitalar ${ }^{6}$.

As complicações secundárias ao AVC podem representar risco à redução da qualidade de vida dos pacientes em função de comprometimento em várias funções motoras, cognitivas e sensoriais; desta forma, o monitoramento é peça chave no plano integral de cuidado ao usuário, sendo essencial na tentativa de detectar precocemente complicações e prevenir perda da autonomia e incapacidade funcional ${ }^{7}$. 
Diante disso, a presente pesquisa apresenta como objetivo principal e norteador evidenciar achados referentes ao monitoramento aos usuários pós-AVC na atenção primária.

\section{MÉTODO}

As buscas por artigos científicos para esta revisão sistemática foram conduzidas por três pesquisadores independentes nas bases de dados eletrônicas MEDLINE (Pubmed), LILACS, SciELO, SCOPUS, WEB OF SCIENCE e BIREME, sem restrição de idioma, período e localização. A pesquisa foi estruturada e organizada na forma PICOS, que representa um acrônimo para População alvo, a Intervenção, Comparação e "Outcomes" (desfechos). População de interesse ou problema de saúde (P) corresponde à pacientes; intervenção (I): pós-AVC; comparação (C), monitoramento; outcome (O): não se aplica (S): estudo transversal, estudo observacional, relatos de caso, estudos de caso-controle, ensaios clínicos controlados, estudos de coorte.

\section{Estratégia de Pesquisa}

Os descritores foram selecionados a partir do dicionário Descritores em Ciências da Saúde (DeCS) e Medical Subject Heading Terms (MeSH) ${ }^{8}$, haja vista a sua grande utilização pela comunidade científica para a indexação de artigos na base de dados PubMed. Diante da busca dos descritores, foi realizada a adequação para as outras bases utilizadas. Foram 
propostas para as buscas os seguintes operadores booleanos: (Rehabilitation) and (stroke) and (speech therapy) and (primary health care). A busca ocorreu em novembro de 2020 de forma concentrada.

\section{Critérios de Elegibilidade}

O desenho dos estudos selecionados para esta revisão sistemática foi do tipo estudo clínico, no entanto não existia restrição quanto ao tipo de estudo avaliado. Foram inclusos estudos sem restrição de idioma, período e localização. Os três estudos admitidos obtiveram pontuação 12 no protocolo modificado ${ }^{9}$ para avaliação da qualidade dos mesmos.

\section{Risco de viés}

A qualidade dos métodos utilizados nos estudos incluídos foi avaliada pelo revisor de forma independente. $A$ avaliação priorizou a descrição clara das informações. Neste ponto, a revisão foi realizada às cegas, mascarando os nomes dos autores e revistas, evitando qualquer viés potencial e conflito de interesses.

\section{Análise dos Dados}

A extração dos dados para o processo de elegibilidade dos estudos foi realizada utilizando-se uma ficha própria elaborada por três pesquisadores em Programa Excel ${ }^{\circledR}$, na qual os dados extraídos foram adicionados por um dos pesquisadores e, então, conferidos por outro pesquisador. Inicialmente foram selecionados de acordo com o título; em 
seguida, os resumos foram analisados e apenas os que fossem potencialmente elegíveis foram selecionados. Com base nos resumos, artigos foram selecionados para leitura integral, foram admitidos os que atendiam a todos os critérios pré-determinados.

\section{Forma de seleção dos estudos}

Inicialmente 0 revisor de elegibilidade foi calibrado para a realização da revisão sistemática. Aqueles que apresentaram um título dentro do âmbito, mas os resumos não estavam disponíveis, também foram obtidos e analisados na íntegra. Foram excluídos estudos fora do âmbito, relatos de caso, cartas ao editor e/ou editorial, revisões de literatura, índices, resumos e estudos em animais. Posteriormente, os estudos elegíveis preliminarmente tiveram o texto completo obtido e avaliado. Em casos específicos, quando o estudo com potencial de elegibilidade apresentasse dados incompletos, os autores poderiam ser contatados por e-mail para mais informações, no entanto esse contato não foi necessário para a presente pesquisa.

\section{Dados Coletados}

Após a triagem, o texto do artigo selecionado foi revisado e extraído de forma padronizada por um autor, identificando-se ano de publicação, local da pesquisa, idioma de publicação, tipo de estudo, amostra, método, resultado e conclusão do estudo. 


\section{Resultado clínico}

O resultado clínico de interesse consistiu em apresentar evidências científicas com base em revisão sistemática da literatura achados referentes ao monitoramento de usuários pós-AVC na atenção primária. Aqueles que não utilizaram a abordagem proposta não fizeram parte da amostra da revisão sistemática.

\section{RESULTADOS E DISCUSSÃO}

Foram identificados 88 artigos inicialmente, dos quais 80 foram excluídos após a leitura dos títulos. Posteriormente, os resumos foram analisados e 6 artigos foram excluídos por não responderem à pergunta norteadora. Procedeu-se a leitura na íntegra dos dois artigos ${ }^{10,11}$ e após a aplicação dos critérios de elegibilidade, ambos foram inclusos na pesquisa (Figura 1). Os estudos analisados foram do tipo ensaio clínico e transversal.

\section{Desenho dos Estudos}

No primeiro estudo ${ }^{10}$, participaram 52 indivíduos compreendendo 24 homens e 28 mulheres, subdivididos em dois grupos, de reabilitação domiciliar dos pacientes (GD; $\mathrm{n}=27$ ) com média de idade de 60,6 anos; e de reabilitação em um centro de saúde primário (CSP; $n=25$ ), com média de idade de 61,7 anos. A função motora foi avaliada por meio da Modified Motor Assessment Scale (MMAS), o equilíbrio postural foi avaliado por meio da Short Form-Postural Assessment Scale for Stroke (SF-PASS) e o nível de 
deficiência dos participantes foi medido por meio do Índice de Reintegração à Vida Normal (IRVN).

Figura 1. Fluxograma PRISMA de busca e análise dos artigos.

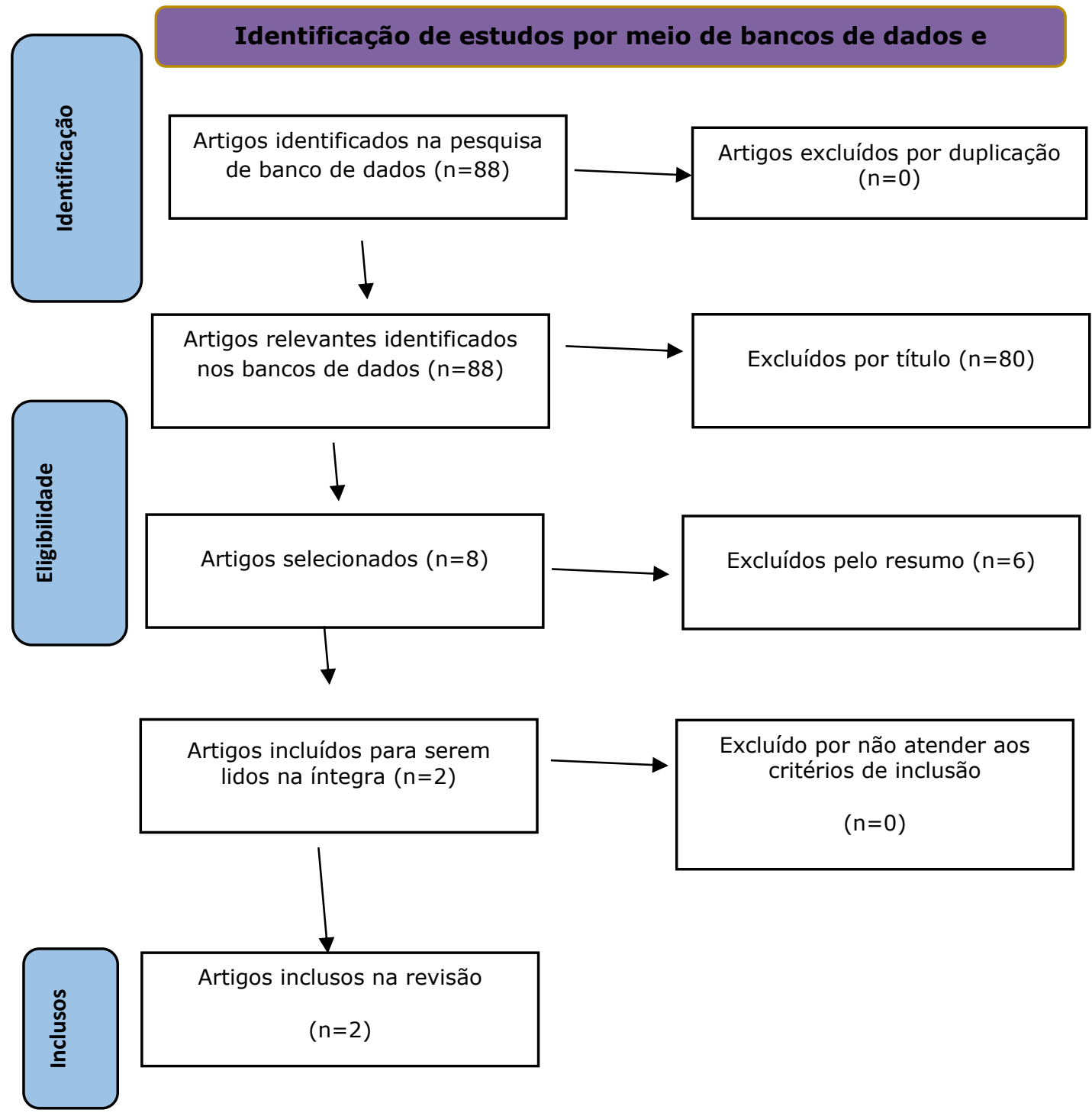


No segundo estudo ${ }^{11}$ os autores tiveram como objetivo verificar os serviços prestados aos pacientes pós-AVC, bem como para estimar a carga de cuidados com o AVC nas unidades públicas de atenção primária. Os participantes foram identificados por meio da revisão do banco de dados de instituições de saúde pública. Foi aplicado o questionário Barthel Index and Brady \& Lawton's IADL scales composto por 77 itens, incluindo prestação atual de serviços para AVC (13), conhecimento sobre o manejo de pacientes após AVC (10), complicações médicas (15), estado funcional e atividades da vida diária (15), cuidados abrangentes de AVC (13), expectativas de melhoria do cuidado (6) e avaliação do cuidador de AVC (5). A última seção era um questionário aberto, que pedia aos entrevistados para escreverem suas opiniões sobre "Qual é o aspecto importante do tratamento do AVC que você acha que é importante na sua prática na atenção primária?".

\section{Principais Achados}

No primeiro estudo, no qual foram inclusos $(n=52)$ participantes, observou-se que o monitoramento foi realizado por meio da assistência fisioterapêutico na atenção primária. Ambos os grupos demonstraram um aumento na velocidade de caminhada desde o início até a semana 10 de acompanhamento, além de um aumento significante nos escores de equilíbrio postural dos participantes do CSP, o que implicou em resultados positivos relacionados à reintegração na comunidade ${ }^{10}$. 
Destaca-se que a integração social é um importante aspecto no processo de reabilitação do AVC, na prevenção à depressão, um importante fator de risco à recuperação física e/ou funcional, além de impactar em questões sociais e familiares ${ }^{11}$.

No segundo estudo, incluindo $(n=121)$ participantes, a maioria dos profissionais $(72,4 \%)$ relataram não ter um plano de cuidados padrão para o gerenciamento de pacientes pós-AVC no nível de atenção primária. Porém, a maioria $(96,6 \%)$ concordou que era necessário ter um plano de cuidados padronizado e isso melhoraria a qualidade do atendimento a esse grupo de pacientes ${ }^{12}$.

Os principais problemas relatados referiam-se às questões de transferência de cuidados, seja para o cuidado domiciliar ou o monitoramento na atenção primária pós alta hospitalar, que diz respeito à longitudinalidade do cuidado. As dificuldades no referenciamento para a reabilitação do paciente refletem no comprometimento do manejo precoce e em medidas de acompanhamento mais eficientes.

Os três tipos mais comuns de encaminhamentos foram a transferência do tipo de atendimento sem acompanhamento adicional pela equipe de setor terciário $(88,1 \%)$, atendimento compartilhado entre os setores terciário e primário $(67,8 \%)$ seguido por pacientes de cuidados primários "internos" que desenvolveram AVC enquanto estavam sob gestão de cuidados primários $(64,4 \%)$. As três primeiras classificações de serviços utilizados no tratamento de pacientes pós-AVC durante os 
cuidados primários são Fisioterapia, Nutricionista e Fonoaudiologia ${ }^{12}$.

Tendo em a importância da reabilitação precoce pósAVC na tentativa de minimizar os possíveis déficits e complicações, as linhas de cuidado ao AVC necessitam ser melhor estruturadas na gestão do cuidado ${ }^{13}$.

As características principais das pesquisas selecionadas para esse estudo, encontram-se expostas na Tabela 1.

\section{CONCLUSÃO}

Os principais achados através da resolutividade das ações pós alta hospitalar encontram-se na fragmentação da comunicação, que impedem a atenção primária como ordenadora do cuidado e a principal responsável pela longitudinalidade da atenção. A eficácia de um plano de atenção integrada para a prestação de serviços de atenção pós-AVC com base na atenção primária deve ser priorizada. Além disso, os estudos mostraram uma evolução significativa dos pacientes que realizaram acompanhamento na atenção primária pós-AVC, demonstrando assim a sua importância de continuidade de intervenção após a alta hospitalar. 
Tabela 1. Síntese dos artigos incluídos.

\begin{tabular}{|c|c|c|c|c|c|}
\hline $\begin{array}{c}\text { Autor/ } \\
\text { Ano/ } \\
\text { Local de } \\
\text { publicaç } \\
\text { ão }\end{array}$ & Objetivo & $\begin{array}{c}\mathbf{N ~ d a} \\
\text { amostra }\end{array}$ & Método & Resultados & Conclusão \\
\hline $\begin{array}{c}\text { Olaleye, } \\
\text { et al. } \\
2013^{10} \\
\text { Nigéria }\end{array}$ & $\begin{array}{l}\text { Comparar os } \\
\text { resultados da } \\
\text { intervenção } \\
\text { fisioterapêutic } \\
\text { a em } \\
\text { pacientes pós- } \\
\text { AVC tratados } \\
\text { no centro de } \\
\text { saúde } \\
\text { primário com } \\
\text { aqueles } \\
\text { tratados em } \\
\text { domicílio. }\end{array}$ & $\begin{array}{c}52 \\
\text { indivíduos } \\
\text { compreend } \\
\text { endo } 24 \\
\text { homens e } \\
28 \\
\text { mulheres }\end{array}$ & $\begin{array}{l}\text { Os participantes foram } \\
\text { aleatoriamente } \\
\text { designados para o grupo } \\
\text { no centro de saúde ( } \mathrm{n} \\
1 / 425 \text { ) ou no grupo } \\
\text { domiciliar (n } 1 / 427 \text { ) e } \\
\text { tratados duas vezes por } \\
\text { semana durante } 10 \\
\text { semanas consecutivas } \\
\text { usando um protocolo de } \\
\text { intervenção } \\
\text { fisioterapêutica. Os } \\
\text { resultados medidos } \\
\text { foram função motora, } \\
\text { equilíbrio e deficiência. }\end{array}$ & $\begin{array}{l}\text { A comparação entre os } \\
\text { grupos não revelou } \\
\text { nenhuma diferença } \\
\text { significante. No entanto, } \\
\text { a comparação dentro do } \\
\text { grupo produziu uma } \\
\text { diferença significante em } \\
\text { cada um dos índices de } \\
\text { recuperação do AVC } \\
\text { medidos ao longo do } \\
\text { período de } 10 \text { semanas } \\
\text { em ambos os grupos. }\end{array}$ & $\begin{array}{c}\text { A intervenção } \\
\text { fisioterapêutica no } \\
\text { centro de atenção } \\
\text { primária à saúde e } \\
\text { respectivas residências } \\
\text { em pacientes pós-AVC } \\
\text { melhorou de forma } \\
\text { semelhante. } \\
\text { O tratamento em } \\
\text { qualquer um desses } \\
\text { locais pode melhorar o } \\
\text { acesso à fisioterapia } \\
\text { após o AVC em uma } \\
\text { comunidade de baixa } \\
\text { renda como a Nigéria. }\end{array}$ \\
\hline $\begin{array}{c}\begin{array}{c}\text { Aziz, et } \\
\text { al. }\end{array} \\
2014^{11} \\
\text { Malásia }\end{array}$ & $\begin{array}{l}\text { Verificar a } \\
\text { prestação de } \\
\text { cuidados pós- } \\
\text { AVC e } \\
\text { problemas } \\
\text { relacionados } \\
\text { entre } \\
\text { Especialistas } \\
\text { em Medicina } \\
\text { da Família que } \\
\text { gerenciam } \\
\text { serviços } \\
\text { públicos de } \\
\text { atenção } \\
\text { primária à } \\
\text { saúde. }\end{array}$ & $\begin{array}{c}121 \\
\text { participant } \\
\text { es }\end{array}$ & $\begin{array}{c}\text { Um questionário } \\
\text { semiestruturado foi } \\
\text { distribuído. } \\
\text { O questionário avaliou os } \\
\text { antecedentes e os } \\
\text { detalhes da prática dos } \\
\text { respondentes, ou seja, } \\
\text { carga estimada de } \\
\text { cuidados com o AVC, } \\
\text { prestação de serviços } \\
\text { atual e opinião sobre a } \\
\text { melhoria do serviço. }\end{array}$ & $\begin{array}{l}\text { Obteve-se uma taxa de } \\
\text { resposta de } 48,8 \% \text {. Para } \\
\text { cada } 100 \text { pacientes } \\
\text { atendidos em centros de } \\
\text { saúde pública a cada } \\
\text { mês, } 2 \text { pacientes têm } \\
\text { acidente vascular } \\
\text { cerebral. O número } \\
\text { médio de pacientes com } \\
\text { AVC atendidos por mês é } \\
5 \text {. } \\
\text { Os pacientes atendidos } \\
\text { foram: alta da atenção } \\
\text { terciária ( } 88,1 \% \text { ), plano } \\
\text { de cuidado } \\
\text { compartilhado com } \\
\text { especialistas ( } 67,8 \%) \text { e } \\
\text { pacientes que } \\
\text { desenvolveram AVC } \\
\text { durante o } \\
\text { acompanhamento na } \\
\text { atenção primária } \\
\text { (64,4\%). } \\
\text { Os encaminhamentos } \\
\text { de frequência foram para } \\
\text { serviços de fisioterapia, } \\
\text { nutricionista e } \\
\text { fonoaudiólogos. }\end{array}$ & $\begin{array}{l}\text { São necessárias } \\
\text { diretrizes de cuidados } \\
\text { pós-alta para AVC e } \\
\text { acesso a serviços de } \\
\text { reabilitação na atenção } \\
\text { primária para pacientes } \\
\text { pós-AVC }\end{array}$ \\
\hline
\end{tabular}




\section{REFERÊNCIAS}

1. Mello GAM, Bridi BPL, Oliveira DC, Jantsch LB. Prevalência de internações hospitalares por acidente vascular cerebral em crianças e adolescentes. Res Soc Dev 2020;9:e45297440. https://doi.org/10.33448/rsd-v9i7.4404

2.Santos LB, Waters C. Perfil epidemiológico dos pacientes acometidos por acidente vascular cerebral: revisão integrativa. Braz J Dev 2020;6:2749-75. https://doi.org/10.34117/bjdv6n1-198

3. Ribeiro PW. O efeito da reperfusão cerebral na deglutição de indivíduos após Acidente Vascular Cerebral (Tese). Botucatu: Universidade Estadual Paulista "Júlio de Mesquita Filho", 2017, 30p.

4.Santana NM, Santos Figueiredo FW, Melo Lucena DM, Soares FM, Adami F, Cardoso LD, et al. The burden of stroke in Brazil in 2016: an analysis of the Global Burden of Disease study findings. BMC Res Notes 2018;11:1-5. https://doi.org/10.1186/s13104-018-3842-3

5.Sarikaya H, Ferro J, Arnold M. Stroke prevention--medical and lifestyle measures. Eur Neurol 2015;73:150-7. https://doi.org/10.1159/000367652

6. Anderle P, Rockenbach SP, Goulart BNGD. Reabilitação pós-AVC: identificação de sinais e sintomas fonoaudiológicos por enfermeiros e médicos da Atenção Primária à Saúde. CoDAS 2019;31. https://doi.org/10.1590/2317-1782/20182018015

7. Morais HCC, Gonzaga NC, Aquino PDS, Araujo TLD. Estratégias de autocuidado apoiado para pacientes com acidente vascular cerebral: revisão integrativa. Rev Esc Enferm USP 2015;49:136-43. https://doi.org/10.1590/S0080-623420150000100018

8. Moher D, Shamseer L, Clarke M. Preferred reporting items for systematic review and meta-analysis protocols (PRISMA-P) 2015 statement. Syst Rev 2015;4:1. https://doi.org/10.1186/2046-4053-41

9.Pithon MM, Santos ALI, Baião FC, Santos RL, Coqueiro RS, Maia LC. Assessment of the effectiveness of mouthwashes in reducing cariogenic biofilm in orthodontic patients: A systematic review. J Dent 2015;43:297-308. https://doi.org/10.1016/j.jdent.2014.12.010

10. Olaleye OA, Hamzat TK, Owolabi MO. Stroke rehabilitation: should physiotherapy intervention be provided at a primary health care centre or the patients' place of domicile? Disabil Rehabilit 2014;36:49-54. https://doi.org/10.3109/09638288.2013.777804

11.Baseman S, Fisher K, Ward L, Bhattacharya A. The relationship of physical function to social integration after stroke. J Neurosci Nurs 2010;42:237-44. https://doi.org/10.1097/JNN.0b013e3181ecafea

12.Aziz AFA, Nordin NAM, Abd Aziz N, Abdullah S, Sulong S, Aljunid SM. Care for post-stroke patients at Malaysian public health centres: self-reported practices of family medicine specialists. BMC Fam Pract 2014;15:40. https://doi.org/10.1186/1471-2296-15-40

13.Dworzynski K, Ritchie G, Playford ED. Stroke rehabilitation: longterm rehabilitation after stroke. J Clin Med 2015;15:461. https://doi.org/10.7861/clinmedicine.15-5-461 